\title{
Relationship of Serum Apolipoprotein A-V Levels, Oxidative Stress and Inflammatory Biomarkers with Hypertriglyceridemia in Type 2 Diabetes Mellitus
}

\author{
Devesh Sharma, ${ }^{1}$ Seema Garg,,$^{1,}$ Mohit Mehndiratta, ${ }^{1}$ S V Madhu, ${ }^{2}$ and Dinesh Puri ${ }^{1}$ \\ ${ }^{1}$ Departement of Biochemistry, University College of Medical Sciences and GTB Hospital, University of Delhi, Delhi, India \\ ${ }^{2}$ Department of Medicine, University College of Medical Sciences and GTB Hospital, University of Delhi, Delhi, India \\ "Corresponding author: Seema Garg, Department of Biochemistry, University College of Medical Sciences, Dilshad Garden, Delhi. Tel: +011-225829745206, E-mail: \\ seegarg@yahoo.com
}

Received 2016 December 15; Revised 2017 February 28; Accepted 2017 April 08.

\begin{abstract}
Background: Serum levels of triglycerides (TGs) are often found to be raised in type 2 diabetes mellitus (T2DM). TG levels $\geq 2.2 \mathrm{mM}$, systemic inflammation and oxidative stress (OS) are known to increase the risk of incident cardiovascular disease (CVD) substantially. In recent years, apolipoprotein A-V (Apo A-V protein) has attracted considerably as a modulator of circulating TG levels.

Objectives: The study was conducted in order to evaluate the levels of Apo A - V proteins and markers of inflammation and OS in patients of T2DM with and without hypertriglyceridemia (HTG) and also to assess correlation between them.

Methods: T2DM patients were categorized into two groups of 40 participants, according to criteria for risk of CVD: group 1/ controls (TG $\leq 1.65 \mathrm{mM}, \mathrm{n}=40$ ) and group 2/ cases (TG $\geq 2.2 \mathrm{mM}, \mathrm{n}=40$ ). Despite the routine investigations, serum levels of Apo A-V, interleukin-6 (IL-6) and Insulin were estimated using ELISA, free fatty acids (FFA) with fluorometric assay and malondialdehyde (MDA) was measured using a spectrophotometer. Comparison of levels and correlation between variables was carried out with appropriate statistical tools.

Results: Serum Apo A-V protein levels were found significantly lower $(\mathrm{P}=0.04)$ and MDA was significantly higher $(\mathrm{P}=0.049)$ in cases. MDA correlated with TG levels positively $(\mathrm{P}=0.000)$ and negatively with high density lipoproteins $(\mathrm{HDL})(\mathrm{P}=0.000)$. However Apo A-V protein levels did not correlate with TG levels $(P=0.819, r=-0.027)$, IL-6 $(r=0.135, P=0.269), F F(r=0.128, P=0.277)$ and MDA $(r$ $=-0.217, \mathrm{P}=0.073)$. IL-6 levels significantly and positively correlated with $\operatorname{HOMA}-\mathrm{IR}(\mathrm{r}=0.327, \mathrm{P}=0.004)$ in the all patients.

Conclusions: In patients of T2DM, low levels of Apo A-V are associated with HTG, indicating that Apo A-V is linked with TG metabolism. Burden of oxidative stress is greater in HTG of T2DM as is evident from MDA levels and its correlation with TG levels. Since oxidative stress is an important patho-physiological basis which increases the risk of CVD in patients of T2DM with HTG. Further studies are required in order to explore the possible role of Apo A-V in TG metabolism in diabetes.
\end{abstract}

Keywords: Type 2 Diabetes, Apolipoprotein A5, Oxidative Stress, Hypertriglyceridemia, Inflammation

\section{Background}

Type 2 diabetes mellitus (T2DM) is a chronic metabolic disorder. The prevalence of this disease is increasing at a very rapid pace throughout the world. At present the disease affects 415 million people worldwide. India is home for second largest number of adults affected with T2DM after China. As per international diabetes federation (IDF) atlas (2016) it is estimated that one in 11 adults have diabetes. In addition every six seconds a person dies from diabetes (1). Complications associated with the disease account for the morbidity and mortality attributed to T2DM. Both of metabolic and vascular complications are observed in patients of T2DM. Oxidative stress along with the state of systemic inflammation has been implicated in the pathophysiological mechanisms which involved in cardiovascular complications in these patients (2). However the ex- act mechanism of development of these complications is not yet completely understood. It is believed that Inflammatory cytokines like interleukin 6 (IL-6) and metabolites like free fatty acids (FFA) have an important role in ensuing vascular complications. Another important contributor to vascular complication is increased serum TG level. Raised TG levels, often found in T2DM, that are assumed to be associated with systemic inflammation (3). They are also believed to be independent risk contributors to coronary artery disease (CAD), a major vascular complication associated with the disease. National cholesterol education program- adult treatment panel -III (NCEP ATP III) criteria suggest that TG levels of $2.2 \mathrm{mM}$ and above moderately increased the risk of CAD (4). The role of TGs in CAD in T2DM is probably associated through increase of oxidative stress which leads to increase the circulating products levels of lipid peroxidation such as malondialdehyde (MDA). 
Modulation of TGs occurs by several mechanisms including hydrolysis by enzyme lipoprotein lipase (LPL). One of the recently discovered players in TG metabolism is Apolipoprotein A-V protein (Apo A-V protein). Many studies have been carried out in order to evaluate correlation between Apo A-V protein and serum TG levels. These studies have been provided contrasting reports. However some studies is found a positive correlation between the two, and others reported negative or no association (5-7). Therefore Apo A-V protein may be an important piece of the puzzle which may give an insight into role of TG's in the pathogenesis of complications in T2DM.

\section{Objectives}

The purpose of this study is to provide a link between TG metabolism, inflammation and oxidative stress in T2DM. In addition this study also aims to evaluate role of Apo A-V in TG metabolism in these patients.

\section{Methods}

\subsection{Study Design and Subjects}

The study was a cross sectional descriptive study and the participants $(n=80)$ included patients of T2DM aged 35 - 60 years visiting the diabetes, endocrine and metabolic centre at University College of Medical Sciences and Guru Teg Bahadur Hospital, New Delhi. The study protocol conformed to the ethical guidelines of the 1975 Declaration of Helsinki and it was approved by institutional ethical committee for human research. The informed consent was obtained from all participants. Diagnosis of T2DM in patients was made based on ADA criteria (8). Then patients were classified according to the NCEP ATP III criteria into two groups (4). Group $1(\mathrm{n}=40)$ : T2DM with $\mathrm{TG}<1.65 \mathrm{mM}$ $(\mathrm{n}=40)$ and Group 2: T2DM with TG $\geq 2.2 \mathrm{mM}(\mathrm{n}=40)$. Group 1 patients were designated as controls and Group 2 as cases. Interviews were conducted in order to collect baseline demographic information and medical history. Thyroid disorders and cardiovascular disease and stroke were assessed from history and examination comprised the exclusion criteria. General physical and systemic examination of all participants was carried out and anthropometric data was recorded. Patients with active infections, renal and hepatic disorders were excluded from the study as were the patients on insulin, hypolipidemic drugs, hormone replacement therapy, thiazide diuretics.

\subsection{Power of Study}

The power of study was calculated by Dalinga et al study to evaluate Apo A-V protein levels in patient with normal and abnormal TG levels (5). In order to detect a difference of $13 \mu \mathrm{g} / 100 \mathrm{~mL}$ in Apo A5 in patients of both groups, the sample size of 40 subjects in each group were sufficient with $80 \%$ power and $95 \%$ confidence level, taking standard deviation of $20 \mu \mathrm{g} / 100 \mathrm{~mL}$ in the both groups.

\subsection{Laboratory Methods}

Blood was collected after overnight fast. Serum was separated within 4 hours, followed by processing for routine biochemical analysis. Aliquots of serum were stored at -80 OC for estimation of serum Apo A-V, IL-6, FFA, insulin and MDA levels. Also plasma was separated from the fluoride vial within 1 hour for glucose estimation. Biochemical parameters (plasma glucose, serum urea, creatinine, transaminases, total bilirubin, total cholesterol (TC), HDL, VLDL and TG) were estimated with system packs on DXC 800 Synchron (Beckman Coulter, USA). HbA1C was evaluated using HPLC (BIORAD D - 10, USA). Fasting levels of Apo A-V protein (BT Biotech, China), IL-6 (Komabiotech, Korea) and insulin (DRG International, USA) was estimated with commercially available ELISA kit following the manufacturer's protocol. Sensitivity of ELISA for Apo A-V estimation was $2.49 \mathrm{ng} / \mathrm{mL}$ and co-efficient of variation (CV) for intra and inter-assay precision was $<8 \%$ and $<10 \%$ respectively. The minimum detectable concentration of Human IL-6 was $<2.1 \mathrm{pg} / \mathrm{mL}$ and intra and inter assay precision for the method was $4.2 \%$ and $6.4 \%$ respectively. For the estimation of serum insulin, within assay variability was $2.6 \%$ and between assay variability was $<6 \%$. Analytical sensitivity of this method was $1.76 \mu \mathrm{IU} / \mathrm{mL}$. FFA was estimated with Fluorometric assay kit (Cayman, USA) as per manufacturer's instructions. Intra-assay CV for this method was 5.9\% and inter-assay CV was 5.6\%.

MDA was estimated spectrophotometrically with thiobarbituric acid (TBA) method (9). The extent of lipid peroxidation was expressed as MDA (nM). Insulin resistance (IR) in the subjects was calculated using the homeostasis model assessment, with the formula:HOMA-IR = fasting plasma glucose $(\mathrm{mM}) \mathrm{x}$ fasting serum insulin $(\mu \mathrm{IU} / \mathrm{mL}) /$ 22.5. HOMA-IR value more than 2.5 was taken as cut off for diagnosing insulin resistance (10).

\subsection{Statistical Analysis}

Data was analyzed using SPSS software, version 20.0 (Illinois, USA). The comparison of variables between two groups was carried out with students' T-test, if data was parametric and Mann Whitney U test for data which was 
non-parametric. In order to evaluate the relationship between different parameters, correlation studies were carried out. Pearson correlation coefficients were calculated where the data was parametric and Spearman rho's correlation was calculated for non-parametric data. Partial regression and bivariate regression was also carried out wherever required. P value less than 0.05 was considered statistically significant.

\section{Results}

\subsection{Demographic Profile of the Patients}

Of the 40 patients in control group, 18 (45\%) were males and $22(55 \%)$ were female, whereas in cases group were 14 (35\%) males and 26 (65\%) females. The clinical and routine biochemical parameters are shown in Table 1. Body mass index (BMI), waist circumference, systolic and diastolic blood pressure, were not significantly different across two groups. Of all patients $(n=80), 12$ were on antihypertensive drugs and all the patients except one, were on oral hypoglycemic agents. 56 patients were prescribed both metformin and glimipride, 18 were prescribed metformin alone and 5 were being managed on vidagliptin. One patient in cases group was not taking any medication and was being managed by diet and exercise.

Hypertension was defined on the basis of recommendations by joint national committee (JNC) VIII blood pressure guideline (11). 7 Individuals (17.5\%) in control group and 9 individuals (22.5\%) in cases group were found hypertensive. Ethnic specific waist circumference as described in IDF criteria for the defining obesity, was used for diagnosing central obesity in participants (12). It was observed that 34 patients ( $85 \%$ ) in control group and in cases group 38 (95\%) patients were obese. Based on HOMA-IR values, it was found that 38 (95\%) individuals were insulin resistant in each group and difference in HOMA IR values was not statistically significant $(\mathrm{P}=0.338)$ (Table 1$)$.

\subsection{Comparative Analysis of the Biochemical Parameters}

Total cholesterol (TC) and TG were found to be significantly higher in cases as compared to controls with $(\mathrm{P}=$ 0.000), HDL-C and LDL-C was almost similar in the both groups ( $\mathrm{P}=0.128$ and 0.238 respectively)(Table 1 ). The mean waist circumference was also not significantly different in two groups $(\mathrm{P}=0.310)$. Levels of serum Apo A-V protein and MDA were compared using students' T-test, and IL-6 and FFA were compared with Mannwhitney U test. Apo A-V protein levels was found to be significantly lower $(\mathrm{P}=0.040)$ in cases as compared to that in controls. MDA levels in cases were significantly higher than in controls. $(\mathrm{P}=0.049)$ (Table 2).

\subsection{Correlational Analysis of the Biochemical Parameters}

As shown in Table 3, a strong positive correlation between MDA and TG levels $(\mathrm{P}=0.000)$ and negative correlation between MDA and HDL levels $(P=0.000)$ was observed in all participants. A strong positive correlation of MDA was also observed with waist circumference (WC) also $(\mathrm{P}=0.008, \mathrm{r}=0.319)$. To assess whether the association with WC was independent of TG or not, bivariate regression analysis was carried out keeping TG as constant. The analysis revealed that correlation of MDA with WC was independent of TG $(\mathrm{B}=-0.133, \mathrm{P}=0.019$, Lower bound: 0.024, upper bound: 242).

Spearman's rho correlation, for non-parametric data was conducted in order to find the association of Apo A-V protein with TG, FFA, IL-6 and MDA in all subjects. Significant correlation was not observed for Apo A-V protein with $\mathrm{TG}(\mathrm{P}=0.819, \mathrm{r}=-0.027)$ and $\mathrm{MDA}(\mathrm{P}=0.073, \mathrm{r}=-0.217)$. In addition no significant correlation of Apo A-V was observed with FFA $(r=0.128, P=0.277)$ and IL-6 $(r=0.135, P=0.269)$. However, it was observed that HOMA-IR correlated significantly with IL-6 $(r=0.288, P=0.014)$ and FFA $(r=0.367$, $\mathrm{P}=0.001)$ in all patients. On conducting partial regression analysis it was observed that association of IL- 6 with HOMA-IR was independent of FFA $(r=0.301, P=0.01)$ and vice versa.

\section{Discussion}

Diabetes mellitus is a complex and progressive disease, burden of which has increased to the extent of causing serious social and economic impact. With 415 million people worldwide, it is no wonder that today diabetes has become a household word (13). Vascular complications in T2DM like CAD, nephropathy, retinopathy are attributed to chronic low grade inflammation which is associated with the disease. Various theories are proposed regarding mechanism of inflammation in T2DM such as its association with increased circulating levels of TGs and FFAs and activation of innate immunity $(3,14)$. Patients of DM have varied derangements in lipid profile particularly TG levels. In addition, incidence of complications is not similar in all patients. Diabetes and HTG are independent risk factors for vascular complications (4). It is evident that the risk of CVD is much higher in individuals who have DM as well as HTG. Thus it is important in order to study the association and the risk of complications according to TG levels in diabetes patients. Therefore, the study was designed with an aim to evaluate the modulators of TG metabolism in patients of T2DM. This study further tried to find out if there is any association of TG and its modulators with markers of inflammation and oxidative stress. 
Table 1. Demographic and Clinical Profile of Study Patients

\begin{tabular}{|c|c|c|c|}
\hline Variables & $\operatorname{Group} 1(n=40)$ & Group $2(n=40)$ & P Value \\
\hline Mean age (years) & $51.17 \pm 10.42$ & $48.68 \pm 10.40$ & 0.294 \\
\hline $\operatorname{BMI}\left(\mathrm{kg} / \mathrm{m}^{2}\right)$ & $28.12 \pm 4.94$ & $28.52 \pm 4.03$ & 0.696 \\
\hline Weight (kg) & $67.88 \pm 13.33$ & $67.07 \pm 12.27$ & 0.782 \\
\hline Waist circumference $(\mathrm{cm})$ & $99.71 \pm 10.93$ & $102.22 \pm 10.57$ & 0.310 \\
\hline Diastolic BP (mm of Hg ) & $80.61 \pm 11.93$ & $84.14 \pm 11.00$ & 0.181 \\
\hline Creatinine $(\mu \mathbf{M})$ & $65.41 \pm 19.44$ & $60.11 \pm 14.14$ & 0.15 \\
\hline Urea (mM) & $9.38 \pm 2.23$ & $9.6 \pm 2.80$ & 0.56 \\
\hline $\operatorname{AST}(\mathbf{I U} / \mathbf{L})$ & $29.5 \pm 14.0$ & $27.38 \pm 7.72$ & 0.67 \\
\hline $\operatorname{ALT}(\mathbf{I U} / \mathbf{L})$ & $34.7 \pm 19.2$ & $28.81 \pm 18.06$ & 0.16 \\
\hline FPG $(\mathbf{m M})$ & $7.79 \pm 3.07$ & $8.53 \pm 2.97$ & 0.287 \\
\hline PPG (mM) & $11.41 \pm 2.83$ & $12.57 \pm 5.05$ & 0.188 \\
\hline $\mathrm{TC}(\mathbf{m M})$ & $4.36 \pm 0.89$ & $5.7 \pm 0.59$ & 0.000 \\
\hline HDL-C (mM) & $8.85 \pm 2.33$ & $9.75 \pm 2.71$ & 0.128 \\
\hline LDL-C (mM) & $29.80 \pm 7.82$ & $32.25 \pm 10.08$ & 0.238 \\
\hline TG $(\mathbf{m M})$ & $5.13 \pm 1.35$ & $13.3 \pm 3.32$ & 0.000 \\
\hline HbA1C (\%) & $7.74 \pm 1.49$ & $5.44 \pm 0.39$ & 0.32 \\
\hline FSI (pM) & $188.0 \pm 115.72$ & $169.21 \pm 65.24$ & 0.939 \\
\hline HOMA-IR & $10.44 \pm 10.13$ & $10.06 \pm 5.27$ & 0.338 \\
\hline
\end{tabular}

Abbreviations: AST, Aspartate Transaminase; ALT, Alanine Transaminase; FPG, Fasting plasma glucose; FSI, Fasting serum insulin; HbA1C, glycated haemoglobin; HOMAIR, Homeostatic model for insulin resistance; HDL-C, High density lipoprotein- cholesterol; LDL-C: Low density lipoprotein- cholesterol; PPG, Post prandial glucose; TC, Total cholesterol; TG, Triglycerides.

${ }^{a}$ values are expressed as mean $\pm S D, P<0.05$ is significant.

Apolipoprotein A-V and HTG in T2DM: The present study reveals that Apo A-V protein was decreased in patients in whom TG level was $\geq 2.2 \mathrm{mM}$. This indicates that Apo A$\mathrm{V}$ protein has some role in affecting TG levels. Previously some studies have been conducted in order to evaluate the role of Apo A-V in TG metabolism in non diabetic individuals $(6,15,16)$. However, some studies have reported mild to moderate positive correlation of Apo A-V protein with TGs; others have observed an inverse relationship between the two parameters. The mechanism of action is proposed for Apo A-V protein by increasing activity of lipoprotein lipase (LPL) and by decreasing VLDL synthesis or secretion $(17,18)$. As a result of this we expected a significant inverse correlation between Apo A-V protein and TG and a positive correlation of Apo A-V with FFA. However, no correlation was observed between Apo A-V and TG concentration in our study. Apo A-V protein and TG levels along with their correlation has been studied in patients of T2DM previously also $(5,19)$. However, none of the studies have categorized the patients on the basis of their TG levels. Nonetheless,
Pruneta-Deloche et al reported that Apo A-V did not correlate with TG in T2DM, a finding similar to our study (19). In addition, we also found that Apo AV protein did not correlate with FFA levels which are a hydrolytic product of TG. The absence of any correlation between Apo A-V and TG as well as FFA levels in our study could be explained on the basis that various other apolipoprotein which contributes to TG metabolism. These include Apo CII and Apo A1 which act as cofactors for LPL and lecithin cholesterol acyltransferase (LCAT) respectively. In addition Apo A-II and C-III are important inhibitors of LPL (20). Dallinga-Thie et al observed that after adjusting for these lipoproteins, contribution Apo A-V in TG metabolism was minimal (5). This supports the findings of our study also. Though we found a low level of Apo $\mathrm{A}-\mathrm{V}$ protein in patients with $\mathrm{HTG}$, a significant relation between Apo A-V and TG was not observed indicating that Apo $\mathrm{A}-\mathrm{V}$ may not be the sole factor in TG modulation at least in diabetes patients.

Inflammatory markers and HTG in T2DM: In this study we did not observe significant difference between IL-6 and FFA 
Table 2. Comparison of Plasma IL-6, FFA and Apo A-V and MDA Levels Among Study Groups ${ }^{\mathrm{a}}$

\begin{tabular}{lcc}
\hline Variables & Group 1 $(\mathbf{n}=\mathbf{4 0})$ & Group 2 $(\mathbf{n}=\mathbf{4 0})$ \\
\hline Apo A-V $(\mathbf{n g} / \mathbf{m L})$ & $466.9 \pm 271.8$ & $369.6 \pm 68.57$ \\
MDA $(\mathbf{n m o l} / \mathbf{m L})$ & $8.01 \pm 3.8$ & 0.040 \\
IL-6 $(\mathbf{p g} / \mathbf{m L})$ & $9.17 \pm 17.52$ & 0.049 \\
FFA $(\mu$ M) & $23.41 \pm 14.21$ & 0.05 .9 \\
\hline
\end{tabular}

${ }^{\mathrm{a}}$ values are expressed as mean $\pm \mathrm{SD}, \mathrm{P}<0.05$ is significant.

Table 3. Correlation of MDA with Lipid Profile Prameters and Anthropometric Parameters in All Subjects

\begin{tabular}{lrr}
\hline Parameter & PValue & R Value \\
\hline TG & 0.001 \\
HDL & 0.001 \\
TG / HDL ratio & 0.001 & -0.444 \\
Waist circumference & 0.000 & 0.005 \\
\hline
\end{tabular}

levels in two groups. There is a possibility that serum IL-6 and FFA levels may not be entirely dependent on TG levels per se. It is well documented that adipose tissue is an important source of these biomarkers (21). Since obesity was equally prevalent in two groups in our study. Therefore, it is not surprising that we did not observe any significantly high levels of IL-6 and FFA in patients with HTG. In addition our study revealed strong positive association of HOMA-IR with FFA and IL-6 levels which indicated that insulin resistance probably is more important factor for systemic inflammation.

Oxidative stress and HTG in T2DM: Oxidative stress has been implicated as culprit in the pathogenesis of complications of diabetes. Hyperglycemia in T2DM leads generation of ROS which reduces oxidative defenses and cause oxidative damage to cell membrane, DNA and cellular proteins (22). MDA levels represents the extent of oxidative damage caused to the lipids yielding the formation of advanced lipoxidation endproducts (ALEs) which leads to oxidative damage (23). In our study we found that MDA levels were significantly high in HTG and were significantly associated with TG levels in all patients. This indicates that TGs contributes to oxidative stress (24) Our findings are similar to the findings of Marcos et al and Al-Aubaidy et al. $(22,25)$. Therefore, we propose that patients of T2DM with HTG may be prone to develop vascular complications owing to their potential to increase oxidative stress. In our study MDA also correlated negatively with HDL thus showing that reduced level of HDL is also associated with oxidative stress. The negative correlation of MDA with HDL in our study indicates the probable antioxidant role/ ac- tion of HDL has been documented previously (20). We also found a strong direct and independent correlation of MDA with waist circumference in all patients. This shows that obesity in T2DM, independently contributes to increase in oxidative stress in the disease. Similar results in non diabetic individuals have been reported by Furukawa et al which is supported our findings (26). These results imply that it is important to control central obesity in order to reduce oxidative stress and inflammation.

In addition, our study also demonstrates a direct positive correlation between HOMA-IR and inflammatory biomarkers namely IL-6 and FFA, which indicates that insulin resistance is associated with systemic inflammation in T2DM as has been demonstrated with previous studies by Festa et al and Arkan et al. (2, 27). However, our study proposes that systemic inflammation is more a function of insulin resistance rather than serum TG levels.

\subsection{Conclusion}

Systemic inflammation and increase in oxidative stress play a key role in giving rise to complication of diabetes. Our study indicates that oxidative stress is higher in patients of T2DM who have HTG. Further, our study reveal for the first time that in T2DM, HTG is associated with lower levels of Apo A-V. This indicates that Apo A-V is linked with modulation of TG levels. Since lowering TG levels may help in reducing complications of diabetes, it is probable that Apo A-V may emerge as an important therapeutic target for altering TG levels. However the role of Apo A-V needs to be further explored. This study re-emphasizes that HTG of T2DM results in increased oxidative stress and insulin resistance contributes to systemic inflammation. Thus it is 
important to control both TG levels and IR in order to minimize the complications of T2DM.

\section{Acknowledgments}

The authors coveys their gratitude to the patients who are participated in the study, the technical staff of department of Biochemistry and hospital lab services. Authors also acknowledge the support of department of Biostatistics and Medical Informatics of our institute.

\section{Footnotes}

Authors' Contribution: Seema Garg, Mohit Mehndiratta, Dinesh Puri conceptualised the study, designed the protocol and supervised the work. Devesh Sharma acquired the data with help from SV Madhu. Devesh Sharma drafted the manuscript and along with Seema Garg, statistically analysed and interepreted the data. Critical review of manuscript was done by Dr. Seema Garg, Dr. Mohit Mehndiratta and Dr. Dinesh Puri. Dr. Dinesh Puri also provided administrative and material support along with Dr. SV Madhu.

Financial Disclosure: Author declares that there is no conflict of interests

Funding/Support: Project was funded by the intramural grant obtained from parent institution, University College of Medical Sciences.

Implications for Health Policy Makers: Controlling TG and insulin resistance may help to decrease the vascular complications which are associated with diabetes by decreasing inflammation and oxidative stress.

\section{References}

1. International Diabetes Federation. . IDF Diabetes Atlas. 7th edn ed. Belgium: Brussels; 2016.

2. Festa A, D’Agostino R, Howard G, Mykkanen L, Tracy RP, Haffner SM Chronic subclinical inflammation as part of the insulin resistance syndrome : The insulin resistance atherosclerosis study (iras). Circulation. 2000;102(1):42-7. doi:10.1161/01.cir.102.1.42.

3. Grunfeld C, Dinarello CA, Feingold KR. Tumor necrosis factor- $\alpha$, interleukin-1, and interferon alpha stimulate triglyceride synthesis in HepG2 cells. Metabolism. 1991;40(9):894-8. doi: 10.1016/00260495(91)90062-2.

4. National Cholesterol Education Program Expert Panel on Detection E, Treatment of High Blood Cholesterol in A. Third Report of the National Cholesterol Education Program (NCEP) Expert Panel on Detection, Evaluation, and Treatment of High Blood Cholesterol in Adults (Adult Treatment Panel III) final report. Circulation. 2002;106(25):3143-421. [PubMed: 12485966].

5. Dallinga-Thie GM, van Tol A, Hattori H, van Vark-van der Zee LC, Jansen $\mathrm{H}$, Sijbrands EJ, et al. Plasma apolipoprotein A5 and triglycerides in type 2 diabetes. Diabetologia. 2006;49(7):1505-11. doi: 10.1007/s00125006-0261-0. [PubMed: 16752169].
6. Ishihara M, Kujiraoka T, Iwasaki T, Nagano M, Takano M, Ishii J, et al. A sandwich enzyme-linked immunosorbent assay for human plasma apolipoprotein A-V concentration.JLipid Res. 2005;46(9):2015-22. doi 10.1194/jlr.D500018-JLR200. [PubMed: 15961791].

7. Pennacchio LA, Olivier M, Hubacek JA, Krauss RM, Rubin EM, Cohen JC. Two independent apolipoprotein A5 haplotypes influence human plasma triglyceride levels. Hum Mol Genet. 2002;11(24):3031-8. [PubMed: 12417524].

8. American Diabetes A. Standards of medical care in diabetes-2013. Diabetes Care. 2013;36 Suppl 1:S11-66. doi: 10.2337/dc13-S011. [PubMed: 23264422].

9. Devasagayam TP, Boloor KK, Ramasarma T. Methods for estimating lipid peroxidation: an analysis of merits and demerits. Indian J Biochem Biophys. 2003;40(5):300-8. [PubMed: 22900323].

10. Matthews DR, Hosker JP, Rudenski AS, Naylor BA, Treacher DF, Turner RC. Homeostasis model assessment: insulin resistance and beta-cell function from fasting plasma glucose and insulin concentrations in man. Diabetologia. 1985;28(7):412-9. [PubMed: 3899825].

11. Armstrong C, Joint National C. JNC8 guidelines for the management of hypertension in adults. Am Fam Physician. 2014;90(7):503-4 [PubMed: 25369633].

12. The IDF consensus worldwide definition of the metabolic syndrome. . [cited April 18]. Available from: http://www.idf.org/webdata/docs/ IDF_Meta_def_final.pdf.

13. International Diabetes Federation. . DF Diabetes Atlas. 6th edn ed. Belgium: Brussels; 2015.

14. Pickup JC, Crook MA. Is type II diabetes mellitus a disease of the innate immune system? Diabetologia. 1998;41(10):1241-8. doi: 10.1007/s001250051058. [PubMed: 9794114].

15. Hodoglugil U, Tanyolac S, Williamson DW, Huang Y, Mahley RW. Apolipoprotein A-V: a potential modulator of plasma triglyceride levels in Turks. J Lipid Res. 2006;47(1):144-53. doi: 10.1194/jlr.M500343JLR200. [PubMed: 16258166].

16. Calandra S, Priore Oliva C, Tarugi P, Bertolini S. APOA5 and triglyceride metabolism, lesson from human APOA5 deficiency. Curr Opin Lipidol. 2006;17(2):122-7. doi: 10.1097/01.mol.0000217892.00618.54. [PubMed: 16531747].

17. Merkel M, Loeffler B, Kluger M, Fabig N, Geppert G, Pennacchio LA, et al. Apolipoprotein AV accelerates plasma hydrolysis of triglyceride-rich lipoproteins by interaction with proteoglycanbound lipoprotein lipase. J Biol Chem. 2005;280(22):21553-60. doi: 10.1074/jbc.M411412200. [PubMed: 15774484].

18. Weinberg RB, Cook VR, Beckstead JA, Martin DD, Gallagher JW, Shelness GS, et al. Structure and interfacial properties of human apolipoprotein A-V. J Biol Chem. 2003;278(36):34438-44. doi: 10.1074/jbc.M303784200. [PubMed: 12810715].

19. Pruneta-Deloche V, Ponsin G, Groisne L, Fruchart-Najib J, Lagarde $\mathrm{M}$, Moulin P. Postprandial increase of plasma apoAV concentrations in Type 2 diabetic patients. Atherosclerosis. 2005;181(2):403-5. doi: 10.1016/j.atherosclerosis.2005.01.034. [PubMed:16039297].

20. Murray RK. Lipid transport and storage. In: Harper's illustrated biochemistry. New York: McGraw-Hill Medical; 2012. pp. 212-23.

21. Shao L, Feng B, Zhang Y, Zhou H, Ji W, Min W. The role of adiposederived inflammatory cytokines in type 1 diabetes. Adipocyte. 2016;5(3):270-4. doi: 10.1080/21623945.2016.1162358. [PubMed: 27617172].

22. Al-Aubaidy HA, Jelinek HF. Oxidative stress and triglycerides as predictors of subclinical atherosclerosis in prediabetes. Redox Rep. 2014;19(2):87-91. doi: 10.1179/1351000213Y.0000000080. [PubMed: 24520969].

23. Wright E Jr, Scism-Bacon JL, Glass LC. Oxidative stress in type 2 diabetes: the role of fasting and postprandial glycaemia. Int $J$ Clin Pract. 2006;60(3):308-14. doi: 10.1111/j.1368-5031.2006.00825.x. [PubMed: 16494646] 
24. Katsuki A, Sumida Y, Urakawa H, Gabazza EC, Murashima S, Nakatan $\mathrm{K}$, et al. Increased oxidative stress is associated with serum levels of triglyceride, insulin resistance, and hyperinsulinemia in Japanese metabolically obese, normal-weight men. Diabetes Care 2004;27(2):631-2. [PubMed: 14747267].

25. Gomez-Marcos MA, Blazquez-Medela AM, Gamella-Pozuelo L, RecioRodriguez JI, Garcia-Ortiz L, Martinez-Salgado C. Serum Superoxide Dismutase Is Associated with Vascular Structure and Function in Hypertensive and Diabetic Patients. Oxid Med Cell Longev.
2016;2016:9124676. doi: 10.1155/2016/9124676. [PubMed: 26635913].

26. Furukawa S, Fujita T, Shimabukuro M, Iwaki M, Yamada Y, Nakajima Y, et al. Increased oxidative stress in obesity and its impact on metabolic syndrome. J Clin Invest. 2004;114(12):1752-61. doi: 10.1172/JCI21625. [PubMed: 15599400].

27. Arkan MC, Hevener AL, Greten FR, Maeda S, Li ZW, Long JM, et al. IKKbeta links inflammation to obesity-induced insulin resistance. Nat Med. 2005;11(2):191-8. doi: 10.1038/nm1185. [PubMed: 15685170]. 